INPLASY

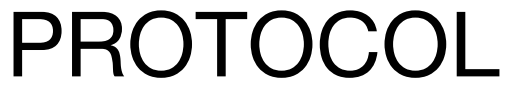

To cite: Shi et al. Meta analysis of efficacy and safety of insulin aspart and biosynthetic human insulin in the treatment of gestational diabetes mellitus. Inplasy protocol 202170047. doi:

10.37766/inplasy2021.7.0047

Received: 15 July 2021

Published: 15 July 2021

Corresponding author: Jinping Shi

903442614@qq.com

Author Affiliation:

Xi'an International Medical

Center Hospital.

Support: Individual funding of research.

Review Stage at time of this submission: The review has not yet started.

Conflicts of interest:

None declared.

\section{Meta analysis of efficacy and safety of insulin aspart and biosynthetic human insulin in the treatment of gestational diabetes mellitus}

Shi, J1; L, F²; X, L3; L, J4; L, X55.

Review question / Objective: The aim of the meta analysis of this randomized controlled trial is to assess the efficacy and safety of insulin aspart in the treatment of gestational diabetes mellitus compared with biosynthetic human insulin. Information sources: We searched PubMed, Cochrane Library, EMBASE database, CNKI database, sinomed database, VIP database and Wannet database for randomized controlled trials of Insulin aspart combined with Biosynthetic human insulin in the treatment of gestational diabetes mellitus from the establishment of the database to July 20,2021

INPLASY registration number: This protocol was registered with the International Platform of Registered Systematic Review and Meta-Analysis Protocols (INPLASY) on 15 July 2021 and was last updated on 15 July 2021 (registration number INPLASY202170047).

\section{INTRODUCTION}

Review question / Objective: The aim of the meta analysis of this randomized controlled trial is to assess the efficacy and safety of insulin aspart in the treatment of gestational diabetes mellitus compared with biosynthetic human insulin.
Condition being studied: Gestational diabetes mellitus (GDM) is the first occurrence or discovery of impaired glucose tolerance during pregnancy. It is one of the common complications of pregnancy. The incidence rate is 4.3\%-5.6\%. A study in 2016 in China 
showed that the incidence rate of gestational diabetes mellitus (GDM) in Beijing area was as high as $19.7 \%$ in 2013. If the blood sugar was not well controlled during pregnancy, the preeclampsia and premature delivery of pregnant women could be caused. Excessive amniotic fluid, cesarean section, postpartum hemorrhage, infection and other adverse outcomes can also lead to neonatal respiratory distress syndrome, jaundice, hypoglycemia and other diseases . Insulin is the first choice to control blood glucose during pregnancy. Insulin aspart does not pass the placental barrier. It is injected subcutaneously immediately before meals, takes effect within 15 minutes, reaches the peak within 31-70 minutes, and lasts for 2-4 hours. Compared with short acting human insulin, insulin aspart has the advantages of better reducing postprandial blood glucose and hypoglycemia before the next meal, without waiting, convenient use and high compliance, It is the first insulin analogue with indications for hyperglycemia during pregnancy in China . A meta-analysis in 2018 showed that insulin aspartate significantly reduced postprandial blood glucose in patients with gestational diabetes mellitus (GDM). However, most of the articles in the meta-analysis did not elaborate on the specific date of blood glucose measurement. The results were heterogeneous, and were not included in maternal and neonatal outcomes. There were more limited. In this study, we searched the published literature and included in the literature that met the criteria of this study for Meta analysis. The aim was to further evaluate the efficacy and safety of insulin aspart and biosynthetic human insulin in the treatment of gestational diabetes mellitus by using evidence-based medicine, so as to provide reference for clinical rational drug use.

\section{METHODS}

Search strategy: Meta analysis was used to evaluate the efficacy and safety of insulin aspart and biosynthetic human insulin in the treatment of gestational diabetes mellitus. Methods We searched PubMed, Cochrane Library, EMBASE database, CNKI database, sinomed database, VIP database and Wannet database for randomized controlled trials of fasudil combined with nimodipine in the treatment of subarachnoid hemorrhage from the establishment of the database to July 20 , 2021.

Participant or population: Women with gestational diabetes mellitus.

Intervention: Treatment with insulin aspart.

Comparator: Treatment with biosynthetic human insulin.

Study designs to be included: RCTs.

Eligibility criteria: In a randomized controlled trial published before July 20, 2021 , the subjects were diagnosed as gestational diabetes mellitus. The study group was treated with insulin aspartate, and the control group was treated with biosynthetic human insulin. The other intervention methods of the two groups were the same.

Information sources: We searched PubMed, Cochrane Library, EMBASE database, CNKI database, sinomed database, VIP database and Wannet database for randomized controlled trials of Insulin aspart combined with Biosynthetic human insulin in the treatment of gestational diabetes mellitus from the establishment of the database to July 20 , 2021.

Main outcome(s): The use of glycated hemoglobin percentage, fasting blood glucose level, postprandial blood glucose level, daily insulin dosage, blood glucose attainment time, insulin resistance index were used to evaluate the effectiveness of treatment. Maternal complications and adverse outcome rates (hypoglycemia, diabetes ketosis, hypertension, preeclampsia, excessive amniotic fluid, cesarean section, premature delivery, etc.) were used. The incidence of neonatal adverse outcomes (respiratory distress, jaundice, hypocalcemia, hypoglycemia, hemocytosis, macrosomia, etc.) was used 
to evaluate the safety of treatment, and the subgroup analysis was performed according to different research data.

Additional outcome (s): Blood homocysteine, insulin resistance index and C-peptide levels before and after treatment.

Data management: Two researchers independently cross screened the eligible articles according to the inclusion criteria and exclusion criteria, The Cochrane evaluation manual was used to evaluate the risk bias of the included studies, and the data were extracted (including researcher, date of publication, number of cases, age, first pregnancy, Singleton pregnancy, drug name, Dosage, Route of administration, Course of administration) In case of disagreement between the two researchers, the third researcher will discuss and decide.

Quality assessment / Risk of bias analysis: According to the Cochrane evaluation manual, two researchers independently cross evaluated the bias risk of the included literatures from seven aspects: whether they were randomly generated, whether they were assigned and hidden, whether they were blinded, whether they were blinded in the outcome evaluation, whether the result data were complete, selective bias and other bias. When the two researchers disagreed, the third researcher discussed and ruled.

Strategy of data synthesis: Revman 5.3 was used to conduct meta-analysis of the included literatures. The binary variable indexes used relative risk (RR) and 95\% confidence interval $(\mathrm{Cl})$ as the statistics of efficacy analysis, and the continuous variable indexes used mean difference (MD) and $95 \%$ confidence interval $(\mathrm{Cl})$ as the statistics of efficacy analysis. The heterogeneity of the included studies was tested by $\mathrm{I}^{2}$. If $\mathbf{P}>\mathbf{0 . 1 0}$ or $\mathrm{I}^{2} \leq \mathbf{5 0 \%}$, the heterogeneity between studies is small, and fixed effect model is used for analysis; If $P \leq \mathbf{0 . 1 0}$ or $I^{2}>\mathbf{5 0 \%}$, the heterogeneity is large. Random effect model was used for analysis, and sensitivity analysis and subgroup analysis were used to determine the source of heterogeneity, Funnel chart was used to detect publication bias.

Subgroup analysis: Subgroups were administered according to different dosages, dosing regimen, age of inclusion, history of diabetes, first pregnancy, and single pregnancy. Subgroups were analyzed according to different dosage, course of treatment, mode of administration, single pregnancy, first pregnancy and combination of drugs.

Sensitivity analysis: Sensitivity analysis was used to observe the size and heterogeneity of the combined effects, and to evaluate the reliability and stability of the results.

Language: Search is not limited by language.

Country(ies) involved: China.

Keywords: Insulin aspart, Biosynthetic human insulin, pregnancy diabetes mellitus, Insulin dosage, Blood glucose target time, Adverse maternal outcomes, Adverse.

Contributions of each author:

Author 1 - Jinping Shi.

Author 2 - Feng, $L$.

Author 3 - Liting, $X$.

Author 4 - Jing, $L$.

Author 5 - Xing, L. 